\title{
Caracteres florales y palinológicos en Acanthostyles (ASTERACEAE, EUPATORIEAE) Y SU RELACIÓN CON LA POLINIZACIÓN
}

\author{
MARIANA A. GROSSI ${ }^{1}$, DIEGO G. GUTIÉRREZ², GONZALO J. MARQUEZ³ y \\ MARÍA LUJÁN LUNA ${ }^{4}$
}

\begin{abstract}
Summary: Floral and palinological characters in Acanthostyles (Asteraceae, Eupatorieae) and its relationship to pollination. Wind pollination in Asteraceae is well documented only in few tribes. Particularly, in Eupatorieae, apparent anemophily has only been reported in 10 species belonging to the genera Critonia, Decachaeta, Eupatorium, Koanophyllon, and Neohintonia, most of which inhabit North and Central America, with only two species from South America. The genus Acanthostyles has only one species, Acanthostyles buniifolius, which is widely distributed in southern South America. This species shows features that may suggest a pollination mechanism mediated by wind. We analyzed in detail some morphological and palynological traits related to the anemophilous syndrome. As a result, we identified some characters of $A$. buniifolius associated to wind pollination: presence of loose and pendulous secondary inflorescence with mainly downward oriented small heads, increased of proportion of stigmatic area in relation to the total length of the style branch, acicular style sweeping laxly arranged and widecaveate, microechinate (= subechinate) pollen. This paper proposes first evidence of possible anemophily for a species of subtribe Dysinaphiinae within the Eupatorieae tribe.
\end{abstract}

Key words: Acanthostyles, anemophily, Austroeupatorium, Eupatorieae, South America.

Resumen: La polinización por el viento en la familia Asteraceae se encuentra documentada en unas pocas tribus. Particularmente en Eupatorieae, sólo se ha reportado aparente anemofilia en 10 especies pertenecientes a los géneros Critonia, Decachaeta, Eupatorium, Koanophyllon y Neohintonia, la mayoría de las cuales crece en América del Norte y Central, con sólo dos especies que alcanzan América del sur. El género Acanthostyles incluye una única especie, Acanthostyles buniifolius, la cual se encuentra ampliamente distribuida en el sur de América del Sur. Esta especie presenta características que sugieren un mecanismo de polinización mediado por el viento. Analizamos en detalle algunos caracteres morfológicos y palinológicos relacionados con el síndrome anemófilo. Como resultado, identificamos algunos atributos asociados con la polinización por el viento: inflorescencia laxa y péndula con capítulos pequeños orientados principalmente hacia abajo, estilo con mayor proporción de área estigmática en relación al largo total de la rama del estilo, pelos barredores del estilo aciculares dispuestos laxamente y polen con amplio cavus, microequinado (=subequinado). Este trabajo aporta las primeras evidencias de la posible anemofilia para una especie de la subtribu Dysinaphiinae, dentro de la tribu Eupatorieae.

Palabras clave: Acanthostyles, anemofilia, Austroeupatorium, Eupatorieae, América del Sur.

\footnotetext{
${ }^{1}$ División Plantas Vasculares, Museo de La Plata, Paseo del Bosque s.n., 1900 La Plata, Argentina. Investigadora CONICET. Email: grossi@fcnym.unlp.edu.ar

2 División Plantas Vasculares, Museo Argentino de Ciencias Naturales (MACN-CONICET), Av. Ángel Gallardo 470, C1405DJR Buenos Aires, Argentina. Investigador CONICET. Email: digutier@macn.gov.ar

${ }^{3}$ Cátedra de Palinología, Facultad de Ciencias Naturales y Museo, Calle 60 y 122, 1900 La Plata, Argentina. Investigador CONICET.

${ }^{4}$ Cátedra de Morfología Vegetal, Facultad de Ciencias Naturales y Museo, Calle 60 y 122, 1900 La Plata, Argentina. CIC-BA.
} 


\section{INTRODUCCIÓN}

La polinización por el viento o anemofilia es una condición derivada en las plantas con flores y ha surgido independientemente a lo largo de su historia evolutiva en numerosas familias (Friedman \& Barrett, 2009). El síndrome anemófilo ha evolucionado al menos 65 veces a partir de ancestros polinizados por animales, y aproximadamente el $10 \%$ de las especies de angiospermas depende de este mecanismo de polinización (Linder, 1998; Ackerman, 2000). Un conjunto de caracteres morfológicos y ecológicos se han asociado a la polinización por el viento en distintos grupos de plantas: inflorescencias péndulas, flores poco llamativas, ausencia de fragancias florales, granos de polen poco ornamentados, entre otros (Wodehouse, 1928; Whitehead, 1969; Grashoff \& Beaman, 1970; Sullivan, 1975; Faegri \& van der Pijl, 1979; Niklas, 1985; Bolick, 1990; Ackerman, 2000; Culley et al., 2002; Friedman \& Barrett, 2009; Mangla \& Gupta, 2015).

La familia Asteraceae, la cual incluye unas 25000 especies, es típicamente entomófila, y representa un componente muy significativo de casi todos los ecosistemas terrestres (Bremer, 1994; Anderberg et al., 2007; Jeffrey, 2009). Sus miembros se caracterizan por presentar principalmente polinización por insectos del orden Hymenoptera (Apoidea) y en menor medida Lepidoptera (Lane, 1996; Mani \& Saravanan, 1999; Anderberg et al., 2007). Numerosas especies de Asteraceae están adaptadas a polinizadores específicos y exhiben intrincados y precisos mecanismos de atracción de los polinizadores, y producción de recompensas (Proctor \& Yeo, 1972; Lane, 1996; Anderberg et al., 2007). Sin embargo, la polinización por el viento ha sido reportada sólo en algunas especies pertenecientes a géneros de distintas tribus de Asteraceae: Anthemideae (i.e. Artemisia L.), Astereae (i.e. Baccharis L.), Eupatorieae (i.e. Critonia P. Browne, Koanophyllon Arruda, Neohintonia R.M. King \& H. Rob., Decachaeta DC and Eupatorium L.), Heliantheae (i.e. Ambrosia L.), Millerieae (i.e. Espeletia Mutis ex Bonpl.) y Senecioneae (i.e. Senecio L.) (Small, 1915; Quintero, 1955; Bianchi et al., 1959; Payne, 1963; Payne \& Svarla, 1970; Grashoff \& Beaman, 1970; Wodehouse, 1971; Sullivan, 1975; Arroyo et al., 1982; Garnock-Jones, 1986; Berry \& Calvo,
1989; Lane, 1996; Watson et al., 2002), las cuales representan líneas evolutivas derivadas dentro de la familia (Funk et. al. 2009; Panero \& Crozier, 2016).

Dentro de este grupo de Asteraceae con ejemplos de anemofilia, se destaca Eupatorieae por ser una de las tribus filogenéticamente más derivadas (Panero \& Crozier, 2016) y diversas, con 16-19 subtribus, alrededor de 180 géneros y unas 20002500 especies (King \& Robinson, 1987; Bremer et al., 1994; Hind \& Robinson, 2007; Robinson et al., 2009). Se distribuye mayormente en el Neotrópico, con algunos taxa en América del Norte y Eurasia (Robinson et al., 2009; Grossi et al., 2013), y tiene su límite meridional de distribución en el norte de la Patagonia en Argentina (Grossi et al., 2011, 2014; Freire, 2014). A pesar de su gran diversidad, la biología floral y los mecanismos de polinización han sido poco estudiados en Eupatorieae (Grashoff \& Beaman, 1970; Sullivan, 1975; Lane, 1996; Torres \& Galetto, 2002, 2007; Cerana, 2004; Lu et al., 2008; Valentin-Silva et al., 2016). El síndrome anemófilo, aunque escaso, ha sido sugerido en 10 especies de la tribu (Grashoff \& Beaman, 1970; Sullivan, 1975), pertenecientes a tres subtribus (Tabla 1). Cabe destacar que dentro de estas especies algunas de ellas presentan un mayor número de caracteres asociados con el síndrome anemófilo, mientras que otras combinan también caracteres relacionados con la entomofilia. Las especies de Eupatorieae, como casi la totalidad de los miembros de Asteraceae, exhiben presentación secundaria de polen, proceso que consiste en la relocalización del mismo durante el desarrollo floral, desde las anteras hacia otros órganos florales, los cuales funcionan posteriormente como presentadores de polen para los agentes polinizadores. En Asteraceae es el estilo a través de sus pelos barredores («sweeping hairs») ubicados en la porción apical estéril del mismo, el que funciona como presentador del polen con una reubicación activa del mismo (Small, 1915; Leins \& Erbar, 1990, 2006; Yeo, 1993; Howell et al., 1993; Ladd, 1994; Erbar \& Leins, 1995).

Estudios sistemáticos y florísticos recientes en el género monoespecífico Acanthostyles R. M. King \& H. Rob. (subtribu Disynaphiinae) muestran preliminarmente indicios de caracteres asociados a una polinización por el viento (Grossi et al., 2011, 2014). Acanthostyles buniifolius (Hook. \& Arn.) R. M. King \& H. Rob. comúnmente conocido como «romerillo», «chilca» o «chirca», es un arbusto 
Tabla 1. Especies de la tribu Eupatorieae previamente reportadas como probablemente anemófilas (Grashoff \& Beaman, 1970; Sullivan, 1975).

\begin{tabular}{|c|c|c|}
\hline Subtribu & Especie & Distribución geográfica \\
\hline \multirow[t]{7}{*}{ Critoniinae } & $\begin{array}{l}\text { Critonia morifolia (Mill.) R.M. King \& H. } \\
\text { Rob. }\end{array}$ & $\begin{array}{l}\text { América central, América del Sur (desde Colombia y } \\
\text { Venezuela hasta Bolivia y Brasil, Estado de Paraná) }\end{array}$ \\
\hline & $\begin{array}{l}\text { Critonia quadrangularis (DC.) R.M. King } \\
\text { \& H. Rob. }\end{array}$ & América central \\
\hline & $\begin{array}{l}\text { Critonia eriocarpa (B.L. Rob. \& Greenm.) } \\
\text { R.M. King \& H. Rob. }\end{array}$ & México \\
\hline & Critonia hebebotrya DC. & México, Guatemala \\
\hline & $\begin{array}{l}\text { Koanophyllon solidaginifolium (A. Gray) } \\
\text { R.M. King \& H. Rob. }\end{array}$ & Sudoeste de EEUU, México \\
\hline & $\begin{array}{l}\text { Koanophyllon solidaginoides (Kunth) } \\
\text { R.M. King \& H. Rob. }\end{array}$ & $\begin{array}{l}\text { América central, América del Sur (desde Colombia hasta } \\
\text { Brasil, Estado de São Paulo) }\end{array}$ \\
\hline & $\begin{array}{l}\text { Neohintonia monantha (Sch. Bip.) R.M. } \\
\text { King \& H. Rob. }\end{array}$ & México \\
\hline \multirow[t]{2}{*}{ Eupatoriinae } & $\begin{array}{l}\text { Eupatorium capillifolium (Lam.) Small ex } \\
\text { Porter \& Britton }\end{array}$ & EEUU, América central \\
\hline & Eupatorium compositifolium Walter & Sudeste de EEUU \\
\hline Hebecliniinae & $\begin{array}{l}\text { Decachaeta incompta (DC.) R.M. King } \\
\text { \& H. Rob. }\end{array}$ & México, Guatemala \\
\hline
\end{tabular}

resinoso, con numerosos capítulos agrupados en cimas paniculiformes laxas y con 5 flores de corolas blancas o rosadas y apéndices estilares largos y pubescentes. Es una de las especies más meridionales de la tribu Eupatorieae. Acanthostyles buniifolius se distribuye en el sudoeste de América del Sur desde el centro de Bolivia hasta el centro de Argentina y se extiende hacia el este en el sur de Brasil, Uruguay y este de Argentina. También habita los sistemas serranos del sur de la provincia de Buenos Aires y de La Pampa, llegando hasta los ambientes asociados al río Negro, como límite sur de su distribución (Grossi et al., 2011, 2014; Fig. 1).

El presente trabajo tiene como objetivo describir caracteres morfológicos florales y polínicos de Acanthostyles que podrían ser indicativos de polinización anemófila.

\section{Materiales y Métodos}

Para estudiar la posible anemofilia en Acanthostyles buniifolius se analizaron los siguientes caracteres morfológicos y palinológicos identificados por autores previos como informativos de los mecanismos de polinización en plantas con flores (Grashoff \& Beaman, 1970; Payne \& Skvarla, 1970; Sullivan, 1975; Friedman \&
Barrett, 2009; Mangla \& Gupta, 2015): posición de la inflorescencia secundaria, orientación del capítulo, proporción entre la longitud total de la rama del estilo y la longitud del área estigmática, características de los pelos de los apéndices estériles de las ramas del estilo, longitud y ancho de las espinas y longitud o alto del cavus del grano de polen. El cavus es un espacio entre las capas de la exina por encima de la capa basal («foot layer») de la ectexina (Blackmore et al., 2009). Para analizar comparativamente los caracteres vinculados con el probable síndrome anemófilo de $A$. buniifolius en relación a una especie entomófila, se seleccionó a Austroeupatorium inulifolium (Kunth) R. M. King \& H. Rob. (Torres \& Galetto, 2002, 2008, 2011), y para todos los caracteres medidos se realizó la prueba estadística no paramétrica de KruskalWallis mediante el programa InfoStat (Di Rienzo et al., 2011) para determinar si las medianas eran significativamente diferentes entre las especies. Todas las mediciones se efectuaron en flores en el mismo estadio de desarrollo con la flor ya abierta y el polen presentado sobre la superficie estigmática.

Se realizaron viajes de campo para la observación de Acanthostyles buniifolius a 13 poblaciones de las provincias argentinas de Buenos Aires, Catamarca, Córdoba, Corrientes, Entre Ríos, Mendoza, La Pampa, Salta y San Luis. Asimismo, se visitó una 


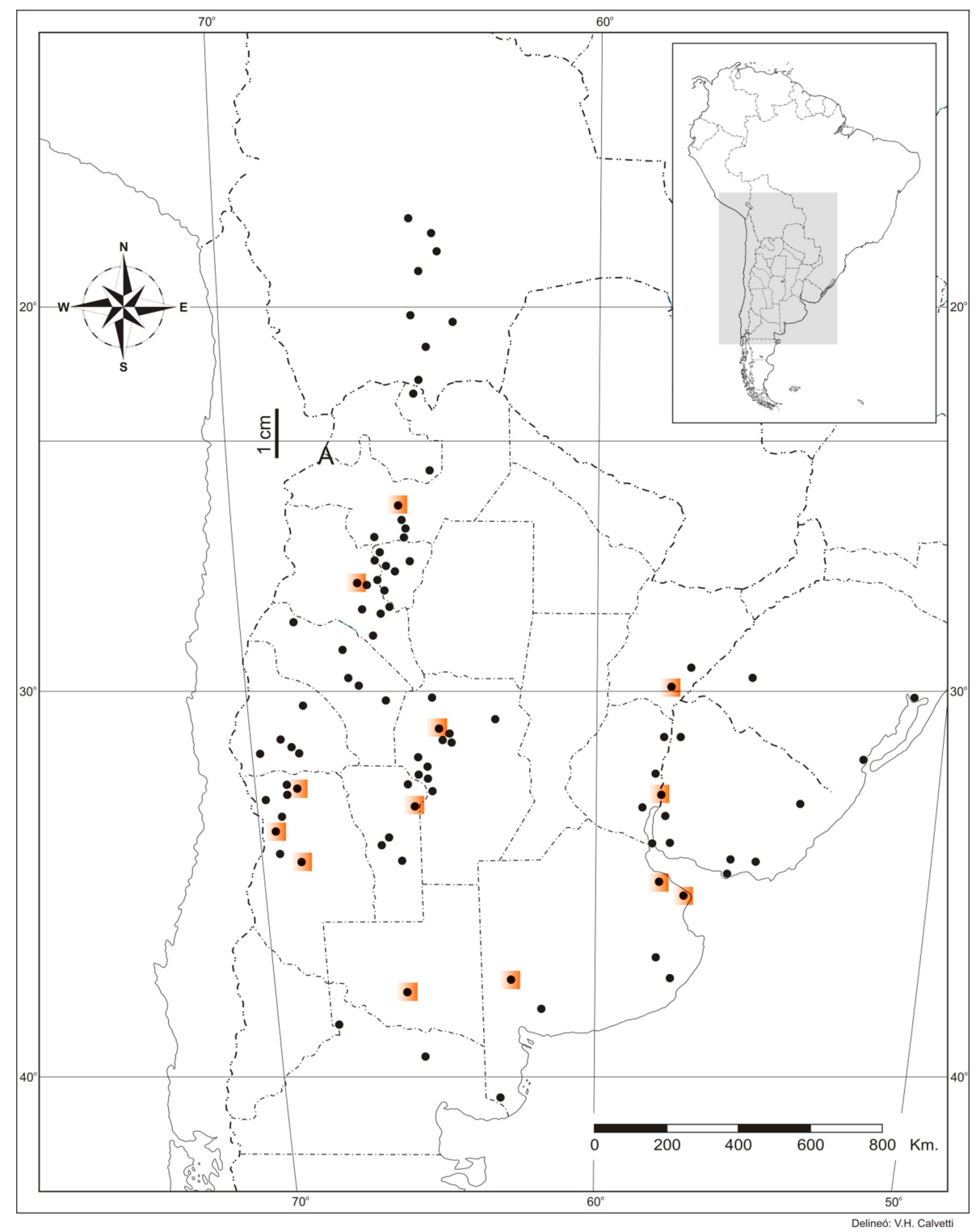

Fig. 1. Acanthostyles buniifolius. Mapa de distribución. Los cuadrados indican las poblaciones visitadas.

población de Austroeupatorium inulifolium en la provincia de Buenos Aires. Los materiales de referencia se encuentran depositados en el Herbario LP (Apéndice 1, Fig. 1).

\section{Inflorescencia y caracteres morfológicos florales}

Para el análisis de la inflorescencia y orientación de los capítulos se observaron a campo distintos individuos sobre transectas en cada localidad. El inicio y la dirección de las mismas fue determinada al azar y las observaciones dentro de cada transecta estuvo separada por 5 a $10 \mathrm{~m}$ de acuerdo al tamaño de la población. Acanthostyles buniifolius florece usualmente en los meses de marzo y abril, aunque en algunas localidades pueden encontrarse individuos, o incluso poblaciones, con capítulos en flor desde noviembre y extenderse su floración hasta el mes de mayo. Las poblaciones en estudio 
fueron visitadas principalmente en los meses de marzo y abril, y algunas de ellas en noviembre y mayo (Apéndice 1). Asimismo, se analizaron especímenes de herbario (herbarios BA, CORD, LP, SI) para complementar las observaciones de campo.

La proporción entre la longitud total de la rama del estilo y la del área estigmática, y las características de los pelos de los apéndices estériles del estilo fueron analizadas tanto en Acanthostyles buniifolius como en Austroeupatorium inulifolium. Las medidas se tomaron en 25 estilos. El diseño del muestreo incluyó seleccionar cinco individuos provenientes de diferentes poblaciones a lo largo de la distribución total de la especie. En cada uno de los cinco individuos se eligieron cinco capítulos de diferente ubicación en la cima paniculiforme, y de cada uno de ellos se seleccionó una única flor (Apéndice 2). Para ello, los mismos fueron previamente hidratados en agua con glicerina, clarificados en hipoclorito de sodio al 5\%, teñidos con safranina al $2 \%$ y montados en portaobjetos. Las medidas y observaciones sobre las ramas del estilo se tomaron en la madurez del mismo, una vez exerto el apéndice estéril de la corola, el cual está incluido en el interior de la misma en los estadios tempranos del desarrollo floral.

Las observaciones y mediciones fueron realizadas en un microscopio óptico marca Nikon Eclipse E200 equipado con una cámara digital. Para la observación de las estructuras al microscopio electrónico de barrido (MEB), las muestras fueron previamente hidratadas en agua con glicerina, fijadas en FAA y, posteriormente, deshidratadas en una serie de alcoholes para ser sometidas a la técnica de punto crítico. Luego fueron montadas sobre tacos y cubiertas con oro-paladio para su observación en un microscopio electrónico de barrido de Alto y Bajo Vacío Marca JEOL, modelo JSM 6360 LV.

\section{Caracteres palinológicos}

En los granos de polen se midió la longitud y ancho de las espinas, y la longitud del cavus. Estas mediciones fueron realizadas sobre 100 granos de polen para cada especie (Acanthostyles buniifolius y Austroeupatorium inulifolium). Dichos granos fueron obtenidos de cinco individuos pertenecientes a cinco poblaciones diferentes. De cada individuo se seleccionó un capítulo y de cada uno de ellos dos flores. De esta manera se analizaron 10 anteras por individuo a partir de las cuales, finalmente, se obtuvieron las medidas de 20 granos de polen. (Apéndice 2). Los granos fueron previamente acetolizados según la técnica convencional (Erdtman, 1986). Las mediciones se efectuaron en un microscopio óptico marca Nikon Eclipse E200 equipado con cámara digital a $100 \mathrm{X}$, colocando directamente los granos sobre el portaobjeto con una gota de glicerina. La morfología polínica fue estudiada mediante microscopía electrónica de barrido (MEB). Los granos fueron montados sobre tacos y cubiertos con oro-paladio para su observación en un microscopio electrónico de barrido de alto y bajo vacío JEOL, JSM 6360 LV. Para la terminología de los granos de polen se siguió a Wodehouse (1928), Erdtman (1952), Punt et al. (1994) y Blackmore et al. 2009.

\section{Resultados}

Inflorescencia y caracteres morfológicos florales

La inflorescencia secundaria de Acanthostyles buniifolius es una cima paniculiforme péndula (Fig. 2A, B), con ramas laxas, capítulos muy numerosos (10-50 por rama florífera), pequeños y elongados (involucro: 3,7-7,6 x 1,8-4,3 mm), orientados generalmente hacia abajo (Fig. 2B). Ocasionalmente en algunas poblaciones se observaron capítulos orientados hacia los costados y, raramente, hacia arriba (Fig. 2C). Estas últimas orientaciones de los capítulos se observaron principalmente en los primeros estadios de desarrollo de la inflorescencia (Fig. 2D) y no fue la tendencia general registrada en la mayoría de las ramas floríferas, individuos o poblaciones. En la especie entomófila Austroeupatorium inulifolium los capítulos, en cimas corimbiformes densas y erectas, siempre están orientados hacia arriba.

Las flores de Acanthostyles buniifolius presentan una corola tubulosa, infundibuliforme, violácea o blanquecina, de 3-5,6 mm de longitud, con tricomas glandulares en su superficie externa; algunas corolas presentan externamente un repliegue a mitad de su longitud (Fig. 3A). Los apéndices estériles de las ramas del estilo sobresalen de la corola cuando ésta madura (Fig. 3B).

La proporción de la longitud total de la 
Bol. Soc. Argent. Bot. 52 (1) 2017

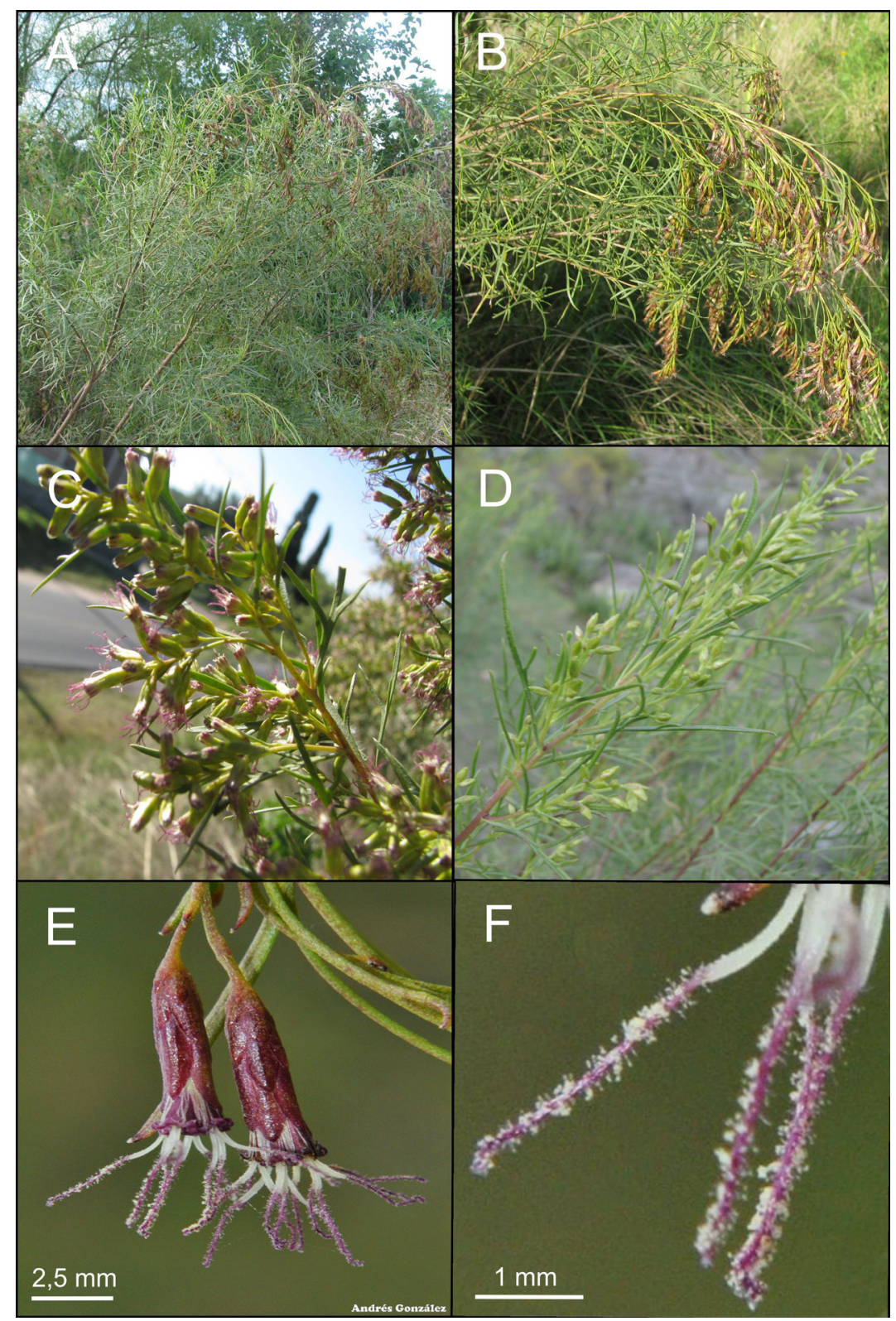

Fig. 2. Acanthostyles buniifolius. A. Aspecto general. B. Inflorescencia secundaria madura, péndula. C. Inflorescencia secundaria mostrando capítulos en distintas orientaciones. D. Inflorescencia secundaria inmadura mostrando capítulos orientados hacia arriba. E. Detalle del capítulo. F. Detalle de las ramas del estilo. (Fotos A y B de M. A. Grossi; C y D de D. G. Gutiérrez; E y F de A. González).

rama del estilo con respecto al área estigmática fue de 1,84 para Acanthostyles buniifolius, evidenciando que gran parte de la superficie de la rama estilar en esta especie corresponde a tejido receptivo (Tabla 2, Fig. 3C). Los pelos barredores del apéndice estéril en A. buniifolius son aciculares, largos (100-120 $\mu \mathrm{m})$ y se disponen laxamente (Fig. 3D, E). En Austroeupatorium inulifolium, la proporción de la longitud total de la rama con respecto al área estigmática resultó 


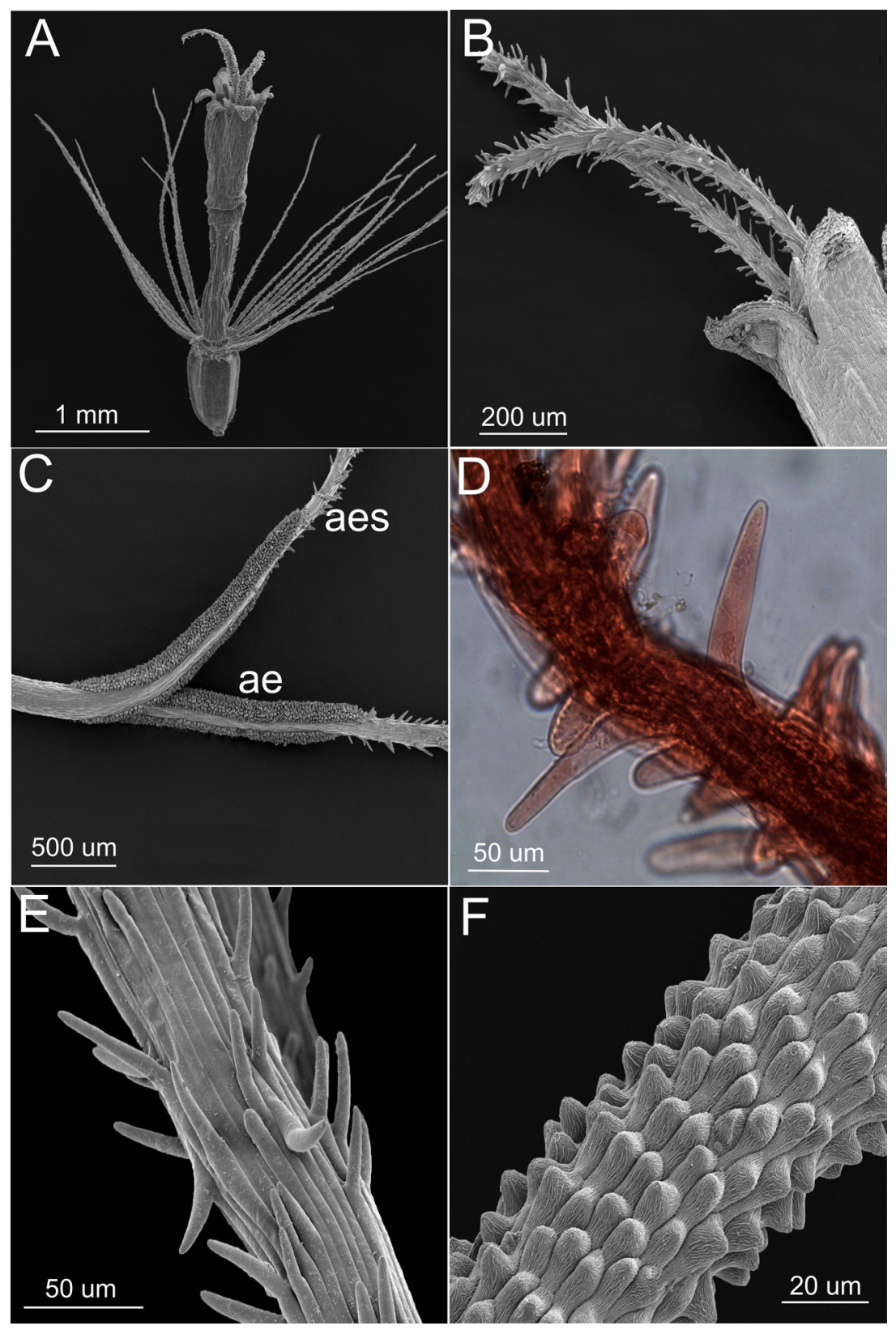

Fig. 3. Acanthostyles buniifolius. A. Flor. B. Ápice de la corola mostrando el apéndice estéril exerto. C. Ramas del estilo mostrando el área estigmática (ae); inicio del apéndice estéril de la rama estilar (aes). D. Pelos barredores del apéndice estéril. E. Detalle de los pelos barredores. F. Austroeupatorium inulifolium: detalle de los pelos barredores del apéndice estéril de las ramas del estilo. A-E: Gutiérrez \& Grossi 1077 (LP, BA); F: Gutiérrez \& Grossi s.n. (LP).

de 4,05. Esto determina una menor superficie de área receptiva (Tabla 2). Los pelos barredores son cortos (10-20 $\mu \mathrm{m})$, redondeados en el ápice y se disponen densamente (Fig. 3F).

\section{Caracteres palinológicos}

El grano de polen de Acanthostyles buniifolius es esférico, con un diámetro polar de 17-20 $\mu \mathrm{m}$ y un diámetro ecuatorial de $17-21 \mu \mathrm{m}$, tricolporado, 
microequinado. La longitud y el ancho de las espinas de la ornamentación de la superficie del grano de polen varió entre 0,80 y $0,95 \mu \mathrm{m}$ x 0,76 y $1,10 \mu \mathrm{m}$, respectivamente (Fig. 4A-C) El tamaño de las espinas resultó significativamente menor que el observado en Austroeupatorium inulifolium (tabla 2, Fig. 4D). En el caso de la longitud del cavus, éste fue significativamente mayor en Acanthostyles buniifolius (Tabla 2).

\section{Discusión}

Los resultados de este trabajo sugieren que Acanthostyles buniifolius reúne caracteres morfológicos tanto florales como palinológicos que se relacionarían con un síndrome anemófilo. Este trabajo constituye el primer reporte de una posible anemofilia en una especie de la subtribu Dysinaphiinae, dentro de la tribu Eupatorieae.

Según trabajos previos (Grashoff \& Beaman, 1970; Mangla \& Gupta, 2015) la presencia de inflorescencias péndulas, con ramas largas y piezas florales orientadas hacia abajo favorecerían la liberación del polen y su posterior transporte por el viento. La inflorescencia secundaria de Acanthostyles buniifolius coincide con los patrones previamente reportados para las especies polinizadas por el viento (Payne, 1963; Whitehead; 1969; Grashoff \& Beaman, 1970; Sullivan, 1975; Faegri \& van der Pijl, 1979; Friedman \& Barrett, 2009).

Las especies anemófilas tienden a aumentar el área receptiva del estigma para asegurar la eficiencia en la captura del polen (Whitehead, 1969; Faegri \& van der Pijl, 1979). En la tribu Eupatorieae esto ha
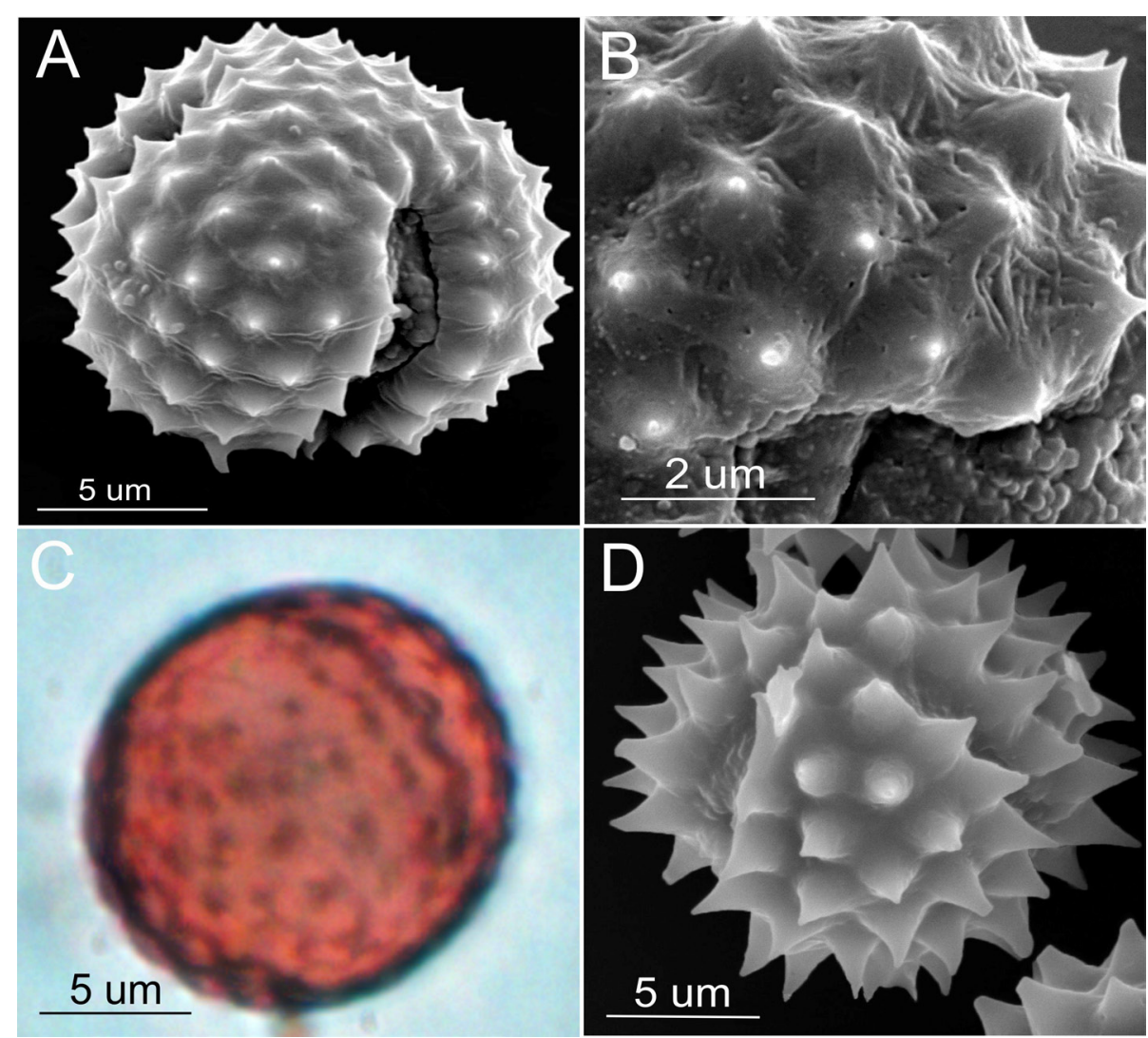

Fig. 4. Acanthostyles buniifolius. A. Grano de polen al microscopio electrónico de barrido (MEB). B. Detalle de las espinas del grano de polen al MEB. C. Grano de polen al microscopio óptico D. Austroeupatorium inulifolium: Grano de polen al MEB. A-C: Gutiérrez \& Grossi 1077 (LP); D: Gutiérrez \& Grossi s.n. (LP). 
sido demostrado por Grashoff \& Beaman (1970) para las especies Decachaeta incompta (DC.) R. M. King \& H. Rob., Koanophyllon solidaginoides (Kunth) R. M. King \& H. Rob. y Neohintonia monantha (Sch. Bip.) R. M. King \& H. Rob., y por Sullivan (1975) para Eupatorium compositifolium Walter. En nuestro trabajo Acanthostyles buniifolius presentó una mayor proporción de superficie estigmática coincidente con lo reportado para otras especies posiblemente anemófilas de la tribu y significativamente mayor comparado con Austroeupatorium inulifolium.

Acanthostyles buniifolius posee un caracterísitico apéndice apical del estilo con pelos barredores aciculares y laxamente distribuidos, único en la tribu (King \& Robinson, 1987; Grossi et al., 2011) (Fig. 3B-E). La disposición laxa de los pelos barredores y la morfología de los mismos podría favorecer la rápida liberación de los granos de polen cuando éstos son expuestos al agente polinizador, al disminuir su retención y adherencia (Grashoff \& Beaman, 1970). En contraposición, los pelos barredores de Austroeupatorium inulifolium son redondeados y cortos, similares a los encontrados en otras especies entomófilas pertenecientes a Eupatorieae (Torres \& Galetto, 2007) y se disponen densamente, lo cual favorecería su adherencia y requeriría la movilización activa de los granos por parte de insectos polinizadores.

El polen equinado ha surgido (presumiblemente de forma independiente) en varias tribus de Asteraceae, principalmente en los linajes más derivados (Blackmore et al., 2009). La reducción del tamaño de las espinas en algunas especies, estaría en consonancia con el síndrome anemófilo (Wodehouse, 1926, 1928; Grashoff \& Beaman, 1970; Faegri \& van der Pijl 1979; Blackmore et al., 2009). De esta manera, las especies anemófilas de Asteraceae poseen polen con espinas reducidas, como en el género Ambrosia (Payne, 1963) y algunas especies de Eupatorium (ej. E. capillifolium; Sullivan, 1975). Acanthostyles buniifolius presentó un grano de polen con espinas considerablemente reducidas menores a $1 \mu \mathrm{m}$ (espínulas de acuerdo a Blackmore et al., 2009) en comparación con especies entomófilas relacionadas. Sullivan (1975) ha sugerido que las espinas ejercen resistencia al transporte por el viento, lo cual podría ser desventajoso para las plantas anemófilas. Sin embargo, otros autores sostienen que la ornamentación del grano de polen tiene sólo una ligera influencia en la dinámica

Tabla 2. Caracteres analizados en Acanthostyles buniifolius y Austroeupatorium inulifolium. Las medidas representan los valores promedio.

\begin{tabular}{|c|c|c|c|c|c|}
\hline Caracteres & $\begin{array}{c}\text { Acanthostyles } \\
\text { buniifolius }\end{array}$ & $\begin{array}{c}\text { Austroeupatorium } \\
\text { inulifolium }\end{array}$ & DE & $\mathbf{p}$ & $\mathbf{H}$ \\
\hline Inflorescencia secundaria & Péndula & Erecta & & & \\
\hline $\begin{array}{l}\text { Orientación de } \\
\text { los capítulos }\end{array}$ & $\begin{array}{c}\text { Generalmente hacia } \\
\text { abajo, a veces hacia } \\
\text { el costado, raramente } \\
\text { hacia arriba }\end{array}$ & Hacia arriba & & & \\
\hline $\begin{array}{l}\text { Proporción longitud total } \\
\text { rama del estilo/longitud } \\
\text { área estigmática }\end{array}$ & 1,84 & 4,05 & 0,22 & $<0,0001$ & 17,28 \\
\hline $\begin{array}{l}\text { Longitud } x \text { ancho de } \\
\text { las espinas del grano } \\
\text { de polen }(\mu \mathrm{m})\end{array}$ & $0,90 \times 0,93$ & $1,87 \times 0,99$ & $0,07-0,11$ & $<0,0001-0,035$ & $29,27-3,19$ \\
\hline $\begin{array}{l}\text { Ancho del cavus del } \\
\text { grano de polen }(\mu \mathrm{m})\end{array}$ & 2,18 & 1,59 & 0,38 & $<0,0001$ & 22,67 \\
\hline $\begin{array}{l}\text { Pelos barredores } \\
\text { ("sweeping hairs") }\end{array}$ & $\begin{array}{l}\text { Laxamente dispuestos, } \\
\text { largos, aciculares }\end{array}$ & $\begin{array}{l}\text { Densamente } \\
\text { dispuestos, cortos, } \\
\text { redondeados }\end{array}$ & & & \\
\hline
\end{tabular}


de vuelo del polen en especies anemófilas, y su función estaría principalmente relacionada con la aglutinación del polen facilitando la polinización entomófila (Faegri \& van der Pijl, 1979; Bolick, 1990). Bolick (1990) considera que las variaciones en la ultraestructura de la pared del grano de polen afectarían la densidad del mismo, influyendo de esta manera en la dinámica de vuelo. En este sentido, se ha reportado que el aumento en el tamaño del cavus del grano de polen podría ser un indicio de anemofilia (Payne \& Skvarla, 1970; Rodríguez Cancelli et al., 2006) al favorecer la flotabilidad del mismo (Harrington \& Metzger, 1963). Los granos de polen con cavus amplio se observan sólo en la subfamilia Asteroideae (en donde se incluye la tribu Eupatorieae), que representa los linajes más derivados dentro de Asteraceae (Blackmore et al., 2009). Acanthostyles buniifolius presenta granos de polen con cavus amplio, y significativamente mayor si se compara con Austroeupatorium inulifolium, lo cual podría estar relacionado también con el síndrome anemófilo. Aún existen controversias sobre el papel que desempeña tanto la ornamentación como la ultraestructura (y la relación entre ambas) como adaptaciones a la anemofilia (Bolick, 1990).

Nuestros resultados sugieren una posible polinización anemófila para Acanthostyles buniifolius, lo cual es sustentado por los siguientes caracteres florales y palinológicos: inflorescencia péndula con ramas laxas y capítulos mayoritariamente orientados hacia abajo, aumento de la proporción del área estigmática en relación al largo total de la rama del estilo, pelos barredores laxamente dispuestos, largos y aciculares, granos de polen con espinas relativamente cortas (espínulas) y cavus amplio.

Una característica de las flores asociada también al síndrome anemófilo es la ausencia de estructuras productoras de recompensas florales (ej. nectarios) (Mangla \& Gupta, 2015). No obstante, las especies de Eupatorieae reportadas como posiblemente anemófilas conservan sus nectarios (Grashoff \& Beaman, 1970). En Acanthostyles buniifolius se están llevando a cabo estudios en este sentido, con el fin de comprobar la ausencia o presencia de estructuras asociadas a recompensas florales.

Dado que el presente trabajo presenta indicios morfológicos compatibles con el síndrome anemófilo en Acanthostyles buniifolius, futuros estudios de ecología de la polinización (como por ejemplo mediante el empleo de trampas de polen) serían necesarios para confirmar la anemofilia en esta especie.

\section{Agradecimientos}

Los autores agradecen a Liliana Katinas y Gisela Sancho por la lectura crítica del manuscrito. Asimismo, a los revisores anónimos quienes han contribuido a mejorar este manuscrito. A los curadores de los herbarios LP, SI y CORD por facilitarnos materiales para llevar a cabo este trabajo. A Cecilia Macluf, Elizabeth Melgarejo Estrada, Esteban Meza Torres, Anibal Prina, Miguel Quijano y Jessica Viera Barreto por su ayuda en los viajes de campo. A Andrés González por facilitarnos la fotografía de Acanthostyles de Uruguay. A Facundo Rodríguez Cravero y Noelia Forte por la edición de las fotografías. A Patricia Sarmiento (MEB-Museo La Plata) y Fabián Tricárico (MEBMACN) por su asistencia técnica. Asimismo los autores agradecen a la Agencia Nacional de Promoción Científica y Tecnológica (ANPCyT), al Consejo Nacional de Investigaciones Científicas y Técnicas (CONICET), y a la Universidad Nacional de La Plata (UNLP) por proveer el financiamiento para la realización de este trabajo.

\section{Bibliografía}

ACKERMAN, J. D. 2000. Abiotic pollen and pollination: ecological, functional, and evolutionary perspectives. Plant Syst. Evol. 222: 167-185.

ANDERBERG, A. A., B. G. BALDWIN, R. G. BAYER, J. BREITWIESER, C. JEFFREY M. O. DILLON, P. ELDENÄS, V. FUNK, N. GARCIA-JACAS, D. J. N. HIND, P. O. KARIS, H. W. LACK G. NESOM, B. NORDENSTAM, C. H. OBERPRIELER, J. L. PANERO, C. PUTTOCK, H. ROBINSON, T. F. STUESSY, A. SUSANNA, E. URTUBEY, R. VOGT, J. WARD \& L. E. WATSON. 2007. Compositae. En: KADEREIT, J.W \& C. JEFFREY (eds.), The families and genera of vascular plants (Series Ed. K. Kubitzki), vol. 3, flowering plants: Eudicots, Asterales. pp. 635. Springer, Berlin.

ARROYO, M. T. K., R. PRIMACK \& J. J. ARMESTO. 1982. Community studies in pollination ecology in the high temperate Andes of central Chile. I. Pollination mechanisms and altitudinal variation. Amer. J. Bot. 69: 82-97. 
BERRY, P. E. \& R.N. CALVO. 1989. Wind pollination, self-incompatibility and shifts in pollination systems in the high Andean genus Espeletia (Asteraceae). Amer. J. Bot. 76: 1602-1614.

BIANCHI, D. E., J. SCHWEMMIN \& W. H. WAGNER. 1959. Pollen release in common ragweed (Ambrosia artemisiifolia). Bot. Gaz. 4: 235-243.

BLACKMORE, S., A. H. WORTLEY, J. J. SKVARLA \& H. ROBINSON. 2009. Evolution of pollen in Compositae. In: FUNK, V. A., A. SUSANNA; T. STUESSY \& R. J. BAYER (eds.), Systematics, Evolution and Biogeography of Compositae, pp. 101-126. International Association for Plant Taxonomy, Vienna.

BREMER, K. 1994. Asteraceae: Cladistics and classification. Timber Press, Portland.

BREMER, K., A. A. ANDERBERG, P. O. KARIS \& J. LUNDBERG. 1994. Tribe Eupatorieae. In: BREMER, K. (ed.), Asteraceae: Cladistics and classification, pp. 625-680. Timber Press, Portland.

BOLICK, M.R. 1990. The pollen surface in wind-pollination with emphasis on the Compositae. In: HESSE, M \& F. EHRENDORFER (eds.), Morphology, development, and systematic relevance of pollen and spores, Plant. Syst. Evol. [Suppl] 5: 39-51. Springer-Verlag, Viena.

CERANA, M. M. 2004. Flower morphology and pollination in Mikania (Asteraceae). Flora 199: 168-177.

CULLEY, T.M., S. G. WELLER \& A. K. SAKAI. 2002. The evolution of wind pollination in angiosperms. Trends Ecol. Evol. 17: 361-369.

DI RIENZO, J.A., F. CASANOVES, M. G. BALZARINI, L. GONZALEZ, M. TABLADA \& C. W. ROBLEDO. 2011. InfoStat. Statistical Software. Grupo Infostat FCA UNC, Córdoba, Argentina.

ERBAR, C. \& P. LEINS. 1995. Portioned pollen release and the syndromes of secondary pollen presentation in the Campanulales-Asterales-complex. Flora 190: 323-338.

ERDTMAN, G. 1952. Pollen Morphology and Plant Taxonomy: Angiosperms: An Introduction to Palynology. Vol. 1. Almqvist \& Wiksell, Stockholm.

ERDTMAN, G. 1986. Handbook of palynology. Munksgaard. Copenhague.

FAEGRI, K. \& L. VAN DER PIJL. 1979. The Principles of Pollination Ecology. 3rd ed. Pergamon, Oxford.

FREIRE, S. E. 2014. Tribu Eupatorieae. En: ZULOAGA, F.O., M. J. BELGRANO \& A. M. ANTON (eds.), Flora Argentina: Flora vascular de la República Argentina, vol. 7(1), pp. 409-435. Estudio Sigma SRL, Buenos Aires.

FRIEDMAN, J. \& S. C. H. BARRETT. 2009. Wind of change: new insights on the ecology and evolution of pollination and mating in wind pollinated plants. Ann. Bot. 103: 1515-1527.
FUNK, V.A., A. SUSANNA, T. F. STUESSY \& H. ROBINSON. 2009. Classification of Compositae. In: FUNK, V.A., A. SUSANNA, T. STUESSY \& R. J. BAYER (eds.), Systematics, evolution and biogeography of Compositae, pp. 171-189. International Association for Plant Taxonomy, Vienna.

GARNOCK-JONES, P. J. 1986. Floret specialization, seed production and gender in Artemisia vulgaris L. (Asteraceae, Anthemideae). Bot. J. Linn. Soc. 92: 285-302.

GRASHOFF, J. L. \& J. H. BEAMAN. 1970. Studies in Eupatorium (Compositae), III. Apparent wind pollination. Brittonia 22: 77-84.

GROSSI, M. A., D. G. GUTIÉRREZ, P. C. BERRUETA \& J. J. MARTÍNEZ. 2011. Acanthostyles (Asteraceae, Eupatorieae): a revision with a multivariate analysis. Aust. Syst. Bot. 24: 87-103.

GROSSI, M.A., L. KATINAS \& . J. NAOKI NAKAJIMA. 2013. Criscianthus, a new genus of Eupatorieae (Asteraceae) with a key to members of the tribe in Africa. Phytotaxa 141: 25-39.

GROSSI, M. A. 2014. Acanthostyles. En: ZULOAGA, F.O., M. J. BELGRANO \& A. M. ANTON (eds.), Flora Argentina: Flora vascular de la República Argentina, vol. 7 , pp. 300-302. Estudio Sigma SRL, Buenos Aires.

HARRINGTON, J. B. \& K. METZGER. 1963. Ragweed pollen density. Am. J. Bot. 50: 532-539.

HIND, D. J. N. \& H. ROBINSON. 2007. Tribe Eupatorieae. In: KADEREIT, J.W. \& C. JEFFREY (eds.), The families and genera of vascular plants (Series Ed. K. Kubitzki), vol. 3, flowering plants: Eudicots, Asterales, pp. 510-574. Springer, Berlin.

HOWELL, G.J., A. T. SLATER \& R. B. KNOX. 1993. Secondary pollen presentation in angiosperms and its biological significance. Aust. .J. Bot. 41: 417-438.

JEFFREY, C. 2009. Evolution of Compositae flowers. In: FUNK, V.A., A. SUSANNA, T. STUESSY \& R. J. BAYER (eds.), Systematics, evolution and biogeography of Compositae, pp. 131-135. International Association for Plant Taxonomy, Vienna.

KING, R. M. \& H. ROBINSON. 1987. The genera of the Eupatorieae (Asteraceae). Monogr. Syst. Bot. Missouri Bot. Gard. 22: 1-581.

LADD, P. G. 1994. Pollen presenters in the flowering plants: form and function. Bot. J. Linn. Soc. 115: 165-195.

LANE, M. A. 1996. Pollination biology of Compositae. In: CALIGARI, P. D. S. \& D. J. N. HIND (eds.), Compositae: biology and utilization. Proceedings of the International Compositae Conference, Kew, 1994, vol. 2, pp. 61-80. Royal Botanic Gardens, Kew. 
LEINS, P. \& C. ERBAR. 1990. On the mechanisms of secondary pollen presentation in the CampanulalesAsterales-complex. Bot. Acta 103: 87-92.

LEINS, P. \& C. ERBAR. 2006. Secondary pollen presentation syndromes of the Asterales - a phylogenetic perspective. Bot. Jahrb. Syst. 127: 83103.

LINDER, H. P. 1998. Morphology and the evolution of wind pollination. In: OWENS, S.J. \& P. J. RUDALL (eds.), Reproductive biology in systematics, conservation and economic botany, pp. 123-135. Royal Botanic Gardens, London.

LU, H., J. SHEN, W. SANG, X. ZHANG \& J. LIN. 2008. Pollen viability, pollination, seed set, and seed germination of Crofton weed (Eupatorium adenophorum) in China. Weed Science 56: 42-51.

MANI, M. S. \& J. M. SARAVANAN. 1999. Pollination ecology and evolution in Compositae (Asteraceae). Science Publishers, Enfield.

MANGLA, Y. \& K. G. GUPTA. 2015. Love in the air: wind pollination ecological and evolutionary considerations. In: KAPOOR, R., I. KAUR \& M. KAUL (eds.), Plant reproductive biology and conservation, $1^{\text {st }}$ ed., pp.234244. IK International, New Delhi.

NIKLAS, K. J. 1985. The aerodynamics of wind pollination. Bot. Rev. 51: 329-383.

PANERO, J. L. \& B. S. CROZIER. 2016 Macroevolutionary dynamics in the early diversification of Asteraceae. Mol. Phylogenet. Evol. 99: 116-132.

PAYNE, W. W. 1963. The morphology of the inflorescence of ragweeds (Ambrosia-Franseria: Compositae). Amer. J. Bot. 50: 872-880.

PAYNE, W.W. \& J. J. SKVARLA. 1970. Electron microscope study of Ambrosia pollen (Compositae: Ambrosieae). Grana 10: 89-100.

PROCTOR, M. C. F. \& P. F. YEO. 1973. The pollination of flowers. Collins, London.

PUNT, W., S. BLACKMORE, S. NILSSON \& A. LE THOMAS. 1994. Glossary of pollen and spores terminology. LPP Foundation, LPP Contributions Series $N^{\circ} 1$, Utrecht.

QUINTERO, J. M. 1955. Cuba. In SAMTER, M. \& O. C. DURHAM (eds.), Regional allergy of the United States, Canada, Mexico and Cuba, pp. 135-142. C. C Thomas, Springfield.

ROBINSON, H., E. SCHILLING \& J. L. PANERO. 2009. Eupatorieae. In: FUNK, V.A., A. SUSANNA, T. STUESSY \& R. J. BAYER (eds.), Systematics, evolution and biogeography of the Compositae, pp. 731-744. International Association for Plant Taxonomy, Vienna.

RODRIGUEZ CANCELLI, R., A. A. SCHNEIDER \& S. G. BAUERMANN. 2006. Morfologia polínica do gênero Pluchea Cass. (Asteraceae), no Rio Grande do Sul, Brasil. Rev. Bras. Pal. 9: 149-156.
SMALL, J. 1915. The pollen-presentation mechanism in the Compositae. Ann. Bot. 29: 457-470.

SULLIVAN, V. I. 1975. Pollen pollination in the genus Eupatorium (Compositae). Canad. J. Bot. 53: 582589.

TORRES, C. \& L. GALETTO. 2002. Are nectar-sugar composition and corolla-tube length related to the diversity of insects that visit Asteraceae flowers? Pl. Biol. 4: 360-366.

TORRES, C. \& L. GALETTO. 2007. Style morphological diversity of some Asteraceae species from Argentina: systematics and functional implications. J. Plant Res. 120: 359-364.

TORRES, C. \& L. GALETTO. 2008. Importancia de los polinizadores en la reproducción de Asteraceae de Argentina central. Acta Bot. Venez. 31: 473-494.

TORRES, C. \& L. GALETTO. 2011. Flowering phenology of co-occurring Asteraceae: a matter of climate, ecological interactions, plant attributes or of evolutionary relationships among species? Org. Divers. Evol. 11: 9-19.

VALENTIN-SILVA, A., M. A. SILVA GODINHO \& M. FARIA VIEIRA. 2016. Life history of Adenostemma brasilianum (Pers.) Cass. (Eupatorieae, Asteraceae): a psychophilous herbaceous species of the Brazilian Atlantic Forest understory. J. Torrey Bot. Soc. 143: 87-92.

WATSON, L.E., P. L. BATES, T. M. EVANS, M. M. UNWIN \& J. R. ESTES. 2002. Molecular phylogeny of subtribe Artemisiinae (Asteraceae), including Artemisia and its allied and segregate genera. $B M C$ Evol. Biol. 2: 17.

WHITEHEAD, D. R. 1969. Wind pollination in the angiosperms: evolutionary and environmental considerations. Evolution 23: 28-35.

WODEHOUSE, R. P. 1926. Pollen grain morphology in the classification of the Anthemideae. Bull. Torrey Bot. Club 53: 479-485.

WODEHOUSE, R. P. 1928. The phylogenetic value of pollen grain characters. Ann. Bot. 42: 891-934.

WODEHOUSE, R. P. 1971. Hayfever plants. 2nd ed. Hafner, New York.

YEO, P. F. 1993. Secondary pollen presentation: form, function and evolution. Pl. Syst. Evol., Suppl. 6: $1-268$.

Recibido el 19 de agosto de 2016, aceptado el 15 de noviembre de 2016. 


\section{Apéndice 1. Poblaciones estudiadas a campo para los análisis de inflorescencias y capítulos}

Acanthostyles buniifolius. ARGENTINA. Prov. Buenos Aires: Pdo. La Plata, ruta 215, Av. 44, entre calles 177 y 179, 8-XI-2014, Gutiérrez \& Grossi 1077 (BA, LP); Pdo. Magdalena, cerca de Ruta 11 y camino a Atalaya, 15-III-2010, Gutiérrez \& Brianese s.n. (LP); Pdo. Tornquist, Sierra de la Ventana, Villa Ventana, 18-IV-2014, Grossi et al. 74 (LP). Prov. Catamarca: Dpto. Andalgalá, Ruta Provincial 18, de Andalgalá a la provincia de Tucumán, 12-V-2008, Gutiérrez et al. 517 (LP). Prov. Córdoba: Dpto. Punilla, Capilla del Monte, 15-IV-2015, Grossi s.n. (LP). ARGENTINA. Prov. Corrientes: Dpto. Mercedes, entre Mercedes y Curuzú Cuatiá, Ruta Nacional 119, km 44, 4-V-2015, Gutiérrez \& Quijano 1075 (BA). Prov. Entre Ríos: Dpto. Colón, Parque Nacional El Palmar, alrededores del camping, XI-2008, Gutiérrez 672 (LP). Prov. Mendoza: Dpto. Las Heras, Ruta 52, antes del ingreso a la reserva, luego de Capdevilla, próximo a termas de Villavicencio, 28-XI-2015, Gutiérrez \& Quijano 1108 (BA), Cacheuta, a $10 \mathrm{~km}$ de las termas de Cacheuta, Ruta 82, 29-XI- 2013, Gutiérrez \& Quijano 1042 (LP); Dpto. San Rafael, Dique Los Reyunos, alrededores del embalse, mirador, 26-XI-2015, Gutiérrez \& Quijano 1100 (BA). Prov. La Pampa: Dpto. Lihuel Calel: Lihuel Calel, 10-III-2016, Grossi et al. s..n. (LP). Prov. San Luis: Dpto. Coronel Pringles, pasando La Florida desde Trapiche a 7,2 km al E del empalme de la Ruta Provincial 9, 10-IV-2008, Gutiérrez et al. 464, 465 (LP). Prov. Salta: Dpto Guachipas, Cuesta del Cebilar, 24-IX-2015, Grossi \& Marquez s.n. (LP).

Austroeupatorium inulifolium. ARGENTINA. Prov. Buenos Aires: Pdo. Ensenada, camino a Isla Santiago, III-2012, Gutiérrez \& Grossi s.n. (LP).

\section{Apéndice 2. Especímenes de herbario utilizados para los estudios de granos de polen y estilos.}

Acanthostyles buniifolius. ARGENTINA. Prov. Buenos Aires: Pdo. La Plata, Ruta 215, Av. 44, entre calles 177 y 179, 8-XI-2014, Gutiérrez y Grossi 1077 (BA, LP); Pdo. Tornquist, Sierra de la Ventana, Villa Ventana, 18-IV-2014, Grossi et al. 74 (LP); Prov. Catamarca: Dpto. Andalgalá, Ruta Provincial 18, de Andalgalá a la provincia de Tucumán, 12-IV-2008, Gutiérrez et al. 517 (LP); Prov. Córdoba: Dpto. Punilla, Capilla del Monte, 15-IV-2015, Grossi s.n. (LP); Prov. La Pampa: Dpto. Lihuel Calel: Lihuel Calel, 10-III2016, Grossi et al. s.n. (LP).

Austroeupatorium inulifolium. ARGENTINA. Prov. Buenos Aires: Pdo. Ensenada, camino a Isla Santiago, III-2012, Gutiérrez \& Grossi s.n. (LP); Prov. Córdoba: Dpto. Río Seco, Ruta 9, a 9 km al N de Villa María, 15-III-1987, Xifreda \& Maldonado 621 (LP); Prov. Jujuy: Dpto. Capital, Quebrada del Jaire, camino a Tiracsi, 20-V-1962, Cabrera et al. 14669 (LP); Prov. Misiones: Dpto. San Javier, Arroyo Portera, camino San Javier-L. N. Além, 13-III-1969, Krapovickas et al. 15256 (LP). BRASIL. Estado Santa Catarina, Azambuja-Brusque, 25-XI-1947, Barbosa Rodriguez 1957 (LP). 
